\title{
Daidzein induces choriocarcinoma cell apoptosis in a dose-dependent manner via the mitochondrial apoptotic pathway
}

\author{
WEI ZHENG ${ }^{1,2}$, TENG LIU $^{3}$, RONG SUN ${ }^{4}$, LEI YANG $^{1}$, RUIFANG AN $^{1}$ and YAN XUE ${ }^{1}$ \\ ${ }^{1}$ Department of Gynecology and Obstetrics, The First Affiliated Hospital of Xi'an Jiaotong University, Xi'an, Shaanxi 710061; \\ ${ }^{2}$ Department of Gynecology and Obstetrics, Ningbo Medical Center Li Huili Eastern Hospital, Ningbo, Zhejiang 315040; \\ ${ }^{3}$ Department of Gynecology and Obstetrics, The Affiliated Hospital of Medical College of Ningbo University, Ningbo, \\ Zhejiang 315020; ${ }^{4}$ Department of Obstetrics and Gynecology, Obstetrics and Gynecology Hospital, \\ Fudan University, Shanghai 200012, P.R. China
}

Received September 3, 2017; Accepted January 26, 2018

\section{DOI: $10.3892 / \mathrm{mmr} .2018 .8604$}

\begin{abstract}
Choriocarcinoma is a malignant gestational trophoblastic disease and relapse or drug resistance occurs in $\sim 25 \%$ of gestational trophoblastic tumors. Cell apoptosis serves a role in the progression from hydatidiform mole to persistent gestational trophoblastic disease. It has been demonstrated that daidzein [7-hydroxy-3-(4-hydroxyphenyl)-4H-chromen4-one] may induce apoptosis in a number of cancer types via the mitochondrial apoptotic pathway by altering the B-cell lymphoma (Bcl)-2/Bcl-2 associated $\mathrm{X}$, apoptosis regulator (Bax) ratio, and activating the caspase cascade. Daidzein also serves a role in regulation of production of human chorionic gonadotropin in trophoblast cells and inhibition of cell proliferation. However, few reports have been published regarding the effect of daidzein on apoptosis in choriocarcinoma. Therefore, in the present study, JAR and JEG-3 human gestational choriocarcinoma cells were used to investigate the effect of daidzein on apoptosis of choriocarcinoma cells. Treatment with daidzein for $48 \mathrm{~h}$ reduced cell viability in a dose-dependent manner. The percentages of early and late apoptotic cells also increased following treatment with daidzein in a dose-dependent manner, with the number of late apoptotic cells increasing more prominently. Furthermore, treatment with daidzein led to apoptosis-associated alterations in nuclear morphology of JAR and JEG-3 cells. Expression levels of cleaved poly(ADP-ribose) polymerase, cleaved caspase-3 and cleaved caspase-9 increased following treatment with daidzein, whereas the $\mathrm{Bcl}-2 / \mathrm{Bax}$ ratio decreased
\end{abstract}

Correspondence to: Professor Yan Xue or Professor Ruifang An, Department of Gynecology and Obstetrics, The First Affiliated Hospital of Xi'an Jiaotong University, 277 Yanta Western Road, Xi'an, Shaanxi 710061, P.R. China

E-mail: xueyan325@126.com

E-mail: ruifangan@163.com

Key words: daidzein, choriocarcinoma, apoptosis, mitochondrial apoptotic pathway in a dose-dependent manner. In conclusion, the results of the present study demonstrate that daidzein may induce apoptosis of choriocarcinoma cells in a dose-dependent manner via the mitochondrial apoptotic pathway.

\section{Introduction}

Gestational trophoblastic disease (GTD) includes a number of pregnancy disorders that arise from placental trophoblastic tissue (1). GTD disorders include, complete and invasive hydatidiform moles, placental site trophoblastic and epithelioid trophoblastic tumors, and choriocarcinoma, which is the most malignant disease, with a tendency to spread to lungs, liver and/or brain (2). The majority of cases of choriocarcinoma occur secondary to a complete mole; however, choriocarcinoma may also occur following any normal or abnormal pregnancy, including partial molar pregnancy, term pregnancy, induced or spontaneous abortion, premature delivery and stillbirth (3). Chemotherapy has notably improved the prognosis of patients with choriocarcinoma in the previous decades; however, $\sim 25 \%$ of gestational trophoblastic tumors are resistant to chemotherapy, or relapse following initial chemotherapy, which may lead to mortality and requires treatment with salvage combination chemotherapy $(4,5)$. During the development of GTD, cell apoptosis serves a role in the progression of a hydatidiform mole into persistent GTD (6-10) and may also influence the chemo-resistance of choriocarcinoma (11). Therefore, novel medicines that are able to induce trophoblastic cell apoptosis may potentially improve efficacy of treatment of choriocarcinoma.

Apoptosis is the primary mechanism of cell death. Generally, there are two main pathways by which apoptosis may be triggered, including the extrinsic receptor-mediated pathway and the intrinsic mitochondrial pathway (12). The extrinsic apoptotic pathway relies on the binding of a death ligand to its membrane receptor on the extracellular domain, including binding of tumor necrosis factor receptor superfamily member 6 (Fas) ligand binding to Fas receptor, and the subsequent formation of a death-inducing signaling complex, leading to the activation of pro-caspase- 8 and promotion of cell death (12-14). In the intrinsic pathway, mitochondria serve 
a role in response to internal stimuli that result in an increase in mitochondrial outer membrane permeability (MOMP) (15). Alterations in MOMP lead to release of proteins from inside the mitochondria to the cytoplasm, and these proteins activate the caspase cascade (typically caspase-9) and other apoptotic responses, including the cleavage of poly(ADP-ribose) polymerase (PARP)-1 (16,17). MOMP is primarily controlled by anti-apoptotic proteins, including apoptosis regulator B cell lymphoma ( $\mathrm{Bcl})-2, \mathrm{Bcl}-2$-like protein 1 , induced myeloid leukemia cell differentiation protein-1, Bcl-2-related protein A1, Bcl-2-like protein 10 and Bcl-2-like protein 2, and pro-apoptotic proteins, including Bcl-2 associated X, apoptosis regulator ( $\mathrm{Bax}), \mathrm{Bcl}-2$ homologous antagonist/killer, BH3-interacting domain death agonist, Bcl-2-like protein 11 and Bcl-2-associated agonist of cell death (18). Therefore, activation of caspase- 3 and cleavage of PARP are markers of apoptosis, whereas the activation of caspase- 9 and reduction of the ratio of Bcl-2 to Bax are considered indicators of activation of mitochondrial apoptotic pathway.

It has recently been proposed that widely accessible and safe dietary ingredients may demonstrate antitumor effects (19). Daidzein (Fig. 1) is classified as isoflavone, and is one of the most commonly ingested and most extensively studied types of phytoestrogen, which is abundant in nuts, fruits, soybeans and soy-based products (20). Previously, daidzein has garnered interest due to its antitumor activity exerted via induction of apoptosis (21). Daidzein induces apoptosis via the mitochondrial apoptotic pathway in a number of cancer types, including breast cancer, gastric carcinoma and hepatic cancer by altering the Bcl-2/Bax ratio and activating the caspase cascade (22-24). Additionally, derivatives of daidzein have been demonstrated to influence apoptosis in colon adenocarcinoma and hepatocellular carcinoma cells $(25,26)$. Jeschke et al (27) observed a significant concentration-dependent decrease in production of human chorionic gonadotropin in trophoblast cells treated with daidzein. Furthermore, the authors previously demonstrated the anti-proliferation activity of daidzein in choriocarcinoma (28). However, few studies regarding the effect of daidzein on apoptosis have been published.

Therefore, the present study aimed to determine whether daidzein may induce choriocarcinoma cell apoptosis via the mitochondrial apoptotic pathway.

\section{Materials and methods}

Cell culture. Human choriocarcinoma cell lines JAR and JEG-3 were obtained from American Type Culture Collection (Manassas, VA, USA) and were cultured in Dulbecco's modified Eagle's medium (Thermo Fisher Scientific, Inc., Waltham, MA, USA) supplemented with $10 \%$ fetal bovine serum (Thermo Fisher Scientific, Inc.) in an atmosphere of $5 \% \mathrm{CO}_{2}$ at $37^{\circ} \mathrm{C}$. Daidzein (Abcam, Cambridge, UK) was dissolved in dimethyl sulfoxide to a concentration of $100 \mathrm{mM}$ and stored at $-20^{\circ} \mathrm{C}$. Daidzein was added into the culture medium at a concentration of $0,25,50$ or $100 \mu \mathrm{M}$ or $48 \mathrm{~h}$ prior to the following experiments.

MTT assay. Cell viability was examined using an MTT assay (Sigma-Aldrich; Merck KGaA, Darmstadt, Germany) following treatment of JAR and JEG-3 cells with 12.5, 25, 50,

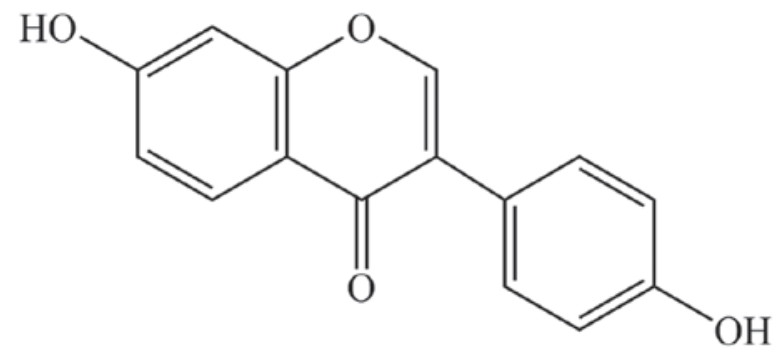

Figure 1. Chemical structure of daidzein

100, 200 and $400 \mu \mathrm{M}$ daidzein for $48 \mathrm{~h}$. The cells were subsequently incubated with $20 \mu 1 \mathrm{MTT}$ solution $(0.5 \mathrm{mg} / \mathrm{ml})$ at $37^{\circ} \mathrm{C}$ for $4 \mathrm{~h}$. The medium was carefully discarded and dimethyl sulfoxide $(150 \mu \mathrm{l})$ was added to dissolve the formazan crystals. The absorbance was measured at a wavelength of $490 \mathrm{~nm}$ using a universal microplate reader (model ELx800; BioTek Instruments, Inc., Winooski, VT, USA).

Cell apoptosis analysis. An Annexin-V-FITC apoptosis detection kit (BD Biosciences, San Jose, CA, USA) was used to determine the apoptotic rate. JAR and JEG-3 cells at $60-80 \%$ confluence were trypsinized, washed with cold PBS twice and resuspended in $100 \mu \mathrm{l}$ binding buffer. Cell suspensions were incubated with Annexin-V $(20 \mu \mathrm{g} / \mathrm{ml})$ and propidium iodide (PI; $50 \mu \mathrm{g} / \mathrm{ml}$ ) for $15 \mathrm{~min}$ at room temperature in the dark and detected using a FACSCalibur flow cytometer (BD Biosciences) and analyzed with FlowJo 7.6.1 software (FlowJo LCC, Ashland, OR, USA). Double negative cells were considered viable, early apoptotic cells were Annexin V positive and PI negative, double positive cells were considered late apoptotic, while Annexin V negative and PI positive cells were necrotic.

Fluorescence microscopy. Cells were fixed in 4\% paraformaldehyde for $15 \mathrm{~min}$ at room temperature, washed with precooled PBS, permeabilized with $0.1 \%$ Triton X-100 for $15 \mathrm{~min}$ and blocked in $1 \%$ bovine serum albumin for $1 \mathrm{~h}$ at room temperature. Cells were subsequently incubated in DAPI solution $(1 \mu \mathrm{g} / \mathrm{ml})$ for $5 \mathrm{~min}$ at room temperature in the dark, and washed again with PBS. Slides were analyzed and images were captured using fluorescent microscopy with an Olympus BX51 microscope at x400 magnification (Olympus Corporation, Tokyo, Japan). A total of 100 cells were randomly selected and the number of cells with apoptotic nuclear morphology were counted; 3 sets of 100 cells were analyzed in each group.

Western blot analysis. Cells were washed once with cold PBS and lysed in radioimmunoprecipitation assay buffer (50 mM Tris, pH 8.0; 150 mM NaCl; 0.1\% SDS; $1 \%$ NP-40; and $0.5 \%$ sodium deoxycholate) containing protease inhibitors. The protein concentration was determined using an enhanced bicinchoninic acid protein assay kit. Protein samples $(20 \mu \mathrm{g} /$ lane $)$ were separated by $8-12 \%$ SDS-PAGE and blotted onto nitrocellulose membranes. The membranes were blocked with $5 \%$ skimmed milk at room temperature for $1 \mathrm{~h}$ and incubated with primary antibodies against $\beta$-actin (cat. no. ab6276; Abcam; 1:2,000), caspase-3 (cat. 
A

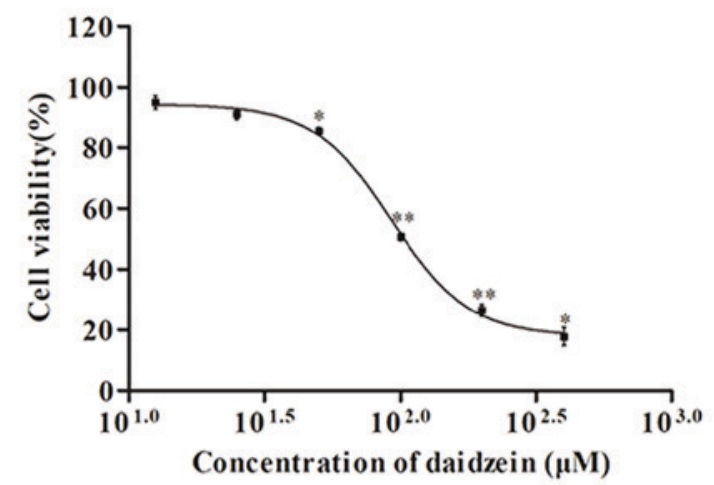

B

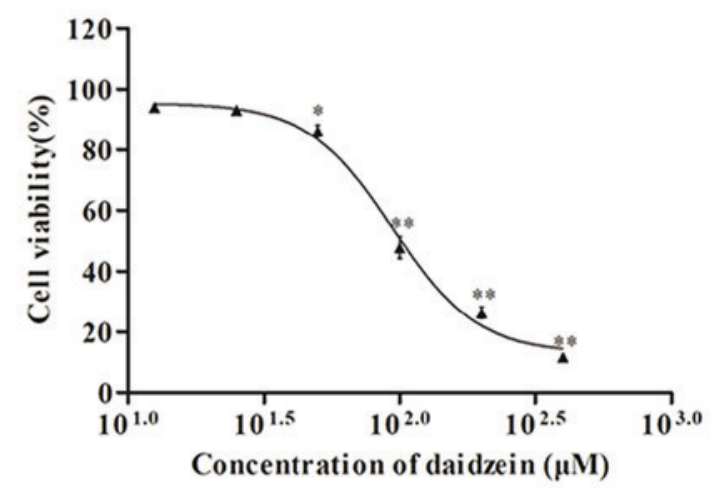

Figure 2. Effect of daidzein on choriocarcinoma cell growth as determined by an MTT assay following treatment for 48 h. Viability of (A) JAR and (B) JEG-3 cells. The results are expressed as the percentage of viable cells compared with the control group $\left(0 \mu \mathrm{M}\right.$ daidzein). ${ }^{*} \mathrm{P}<0.05$ and ${ }^{* * *} \mathrm{P}<0.01$ vs. prior concentration (for example, 50 vs. $25 \mu \mathrm{M}, 100$ vs. $50 \mu \mathrm{M}$ )

no. 9662; Cell signaling Technology, Inc., Danvers, MA, USA; 1:1,000), caspase-9 (cat. no. 9502; Cell signaling Technology, Inc.; 1:1,000), PARP (cat. no. 9532; Cell signaling Technology, Inc.; 1:1,000), Bcl-2 (cat. no. sc-7382; Santa Cruz Biotechnology, Inc., Dallas, TX, USA; 1:500), Bax (cat. no. sc-7480; Santa Cruz Biotechnology, Inc.; 1:500) at $4^{\circ} \mathrm{C}$ overnight. Subsequently, cells were washed in Tris buffered saline containing $0.1 \%$ Tween 20 , and incubated for $1 \mathrm{~h}$ with horseradish peroxidase-conjugated goat anti-rabbit and goat anti-mouse secondary antibodies (cat. nos. KC-RB-035 and KC-MM-035; Aksomics, Inc., Shanghai, China; 1:5,000) at room temperature. Protein bands were visualized with a Molecular Imager ChemiDoc XRS System (Bio-Rad Laboratories, Inc., Hercules, CA, USA) using an enhanced chemiluminescence reagent (EMD Millipore, Billerica, MA, USA). Protein bands were quantified using ImageLab software (version 4.1; Bio-Rad Laboratories, Inc.).

Statistical analysis. All experiments were repeated at least three times. Statistical analysis was carried out using SPSS software package (version 19.0; IBM Corp., Armonk, NY, USA). Data were expressed as the mean \pm standard deviation. Differences between two groups were performed using Student's t-test and multiple comparisons were performed using one-way analysis of variance, followed by Dunnett's test. $\mathrm{P}<0.05$ was considered to indicate a statistically significant difference.

\section{Results}

Daidzein reduces viability of choriocarcinoma cells. The effect of daidzein on viability of choriocarcinoma cell lines JAR and JEG-3 was investigated using the MTT assay. The two cell lines were treated with daidzein at concentrations of $12.5,25,50,100,200$ and $400 \mu \mathrm{M}$. The results demonstrated that daidzein induced a dose-dependent decrease in viability of JAR (Fig. 2A) and JEG-3 (Fig. 2B) cells when the concentration was higher than $25 \mu \mathrm{M}$, with an $\mathrm{IC}_{50}$ of $\sim 100 \mu \mathrm{M}$.

Treatment with daidzein induces apoptosis. To determine whether apoptosis contributed to the reduction in viability of daidzein-treated cells, fluorescence-activated cell sorting analysis was performed to detect apoptosis. The percentages of early and late apoptotic cells increased following treatment with daidzein. In JAR cells, early apoptotic cells accounted for 2.79, $3.61,12.66 \%$ of the cell population following treatment with 25 , 50 and $100 \mu \mathrm{M}$, respectively, whereas the percentage of early apoptotic cells was $2.35 \%$ in the control $(0 \mu \mathrm{M})$ group $(25 \mu \mathrm{M}$, $\mathrm{P}<0.05$ vs. control; 50 and $100 \mu \mathrm{M}, \mathrm{P}<0.01$ vs. control). The percentages of late apoptotic cells following treatment with 0 , 25, 50 and $100 \mu \mathrm{M}$ daidzein were 4.94, 9.4818 .62 and $22.48 \%$ respectively $(25 \mu \mathrm{M}, \mathrm{P}<0.05$ vs. control; 50 and $100 \mu \mathrm{M}, \mathrm{P}<0.01$ vs. control; Fig. 3A and B). The percentage of early apoptotic JEG-3 cells increased from $0.07 \%$ at $0 \mu \mathrm{M}$ to $0.87,1.75$ and $2.89 \%$ at 25,50 and $100 \mu \mathrm{M}$ daidzein, respectively $(\mathrm{P}<0.01)$, while the percentage of late apoptotic cells increased from $1.28 \%$ at $0 \mu \mathrm{M}$ to $6.43,13.05$ and $19.57 \%$ at 25,50 and $100 \mu \mathrm{M}$, respectively $(\mathrm{P}<0.01$; Fig. $3 \mathrm{C}$ and $\mathrm{D})$. The aforementioned results indicated that daidzein induced choriocarcinoma cell apoptosis in a dose-dependent manner. This effect may be due to the larger proportion of late apoptotic cells.

Daidzein alters the morphology of choriocarcinoma cells. Morphological alterations are another manifestation of cell apoptosis. Therefore, following treatment with 0 and $100 \mu \mathrm{M}$ daidzein for $48 \mathrm{~h}$, morphological characteristics of JAR and JEG-3 cells were examined by fluorescence microscopy following DAPI staining. In control cells ( $0 \mu \mathrm{M}$ daidzein), the nuclei appeared round, intact and uniformly stained, and few apoptotic cells were observed. However, following treatment with daidzein, cell nuclei exhibited different degrees of apoptosis, including: i) phase I, where rippled or creased nuclei were observed, with mildly condensed chromatin; ii) phase IIa, where chromatin was highly condensed; and iii) phase $\mathrm{IIb}$, where fragmented nuclei were observed. The number of cells with apoptotic nuclear morphology increased following treatment with daidzein, compared with the untreated control $(\mathrm{P}<0.01$ Fig. 4). The aforementioned results suggested that daidzein leads to alterations in the nuclear morphology of choriocarcinoma cells, thereby further indicating the pro-apoptotic effect of daidzein.

Daidzein affects the protein levels of markers of apoptosis. The levels of apoptosis-associated proteins were detected by 
A

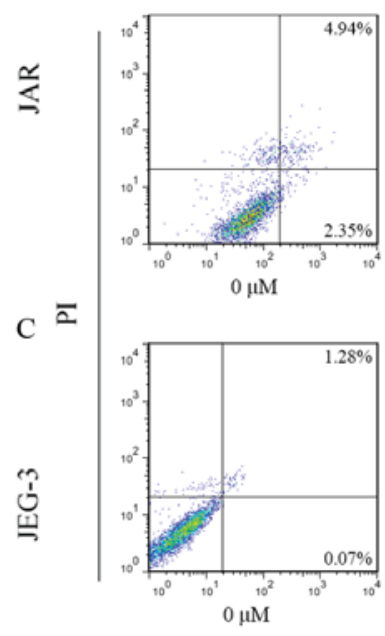

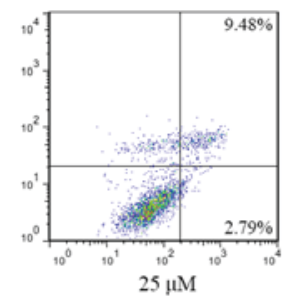

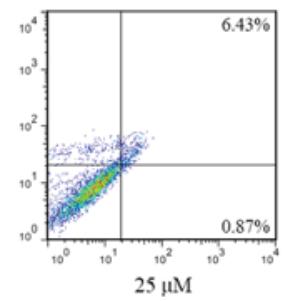

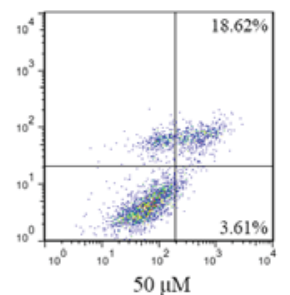

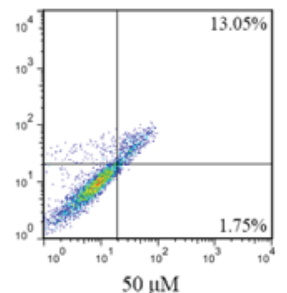

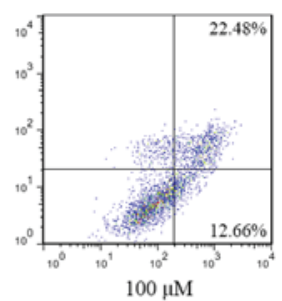

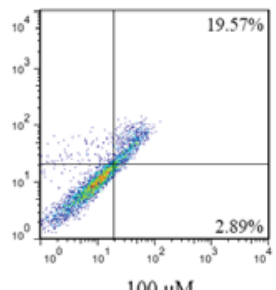

$100 \mu \mathrm{M}$

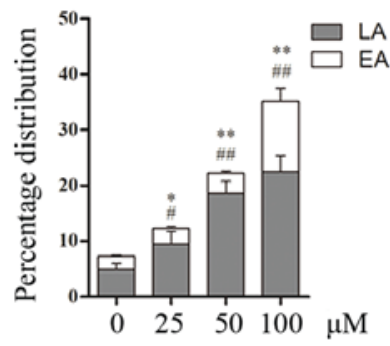

$\mathrm{D}$

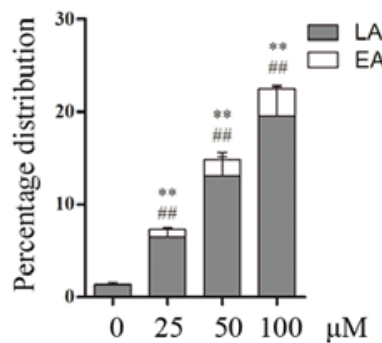

Annexin- $\mathrm{V}$

Figure 3. Effects of different concentrations of daidzein on choriocarcinoma cell apoptosis, as detected by Annexin-V/PI staining and flow cytometry. (A) Representative image (B) quantification and percentages of early and late apoptotic JAR cells, detected by flow cytometry. (C) Representative image and (D) quantification and percentages of early and late apoptotic JEG-3 cells, detected by flow cytometry. ${ }^{*} \mathrm{P}<0.05$ and ${ }^{* *} \mathrm{P}<0.01$ vs. EA in the $0 \mu \mathrm{M}$ group; ${ }^{*} \mathrm{P}<0.05$ and ${ }^{\# \#} \mathrm{P}<0.01$ vs. LA in the $0 \mu \mathrm{M}$ group. PI, propidium iodide; EA, early apoptotic; LA, late apoptotic.
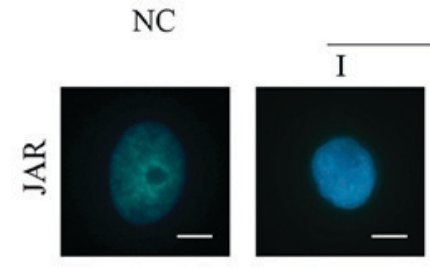

$\mathrm{Dd}$
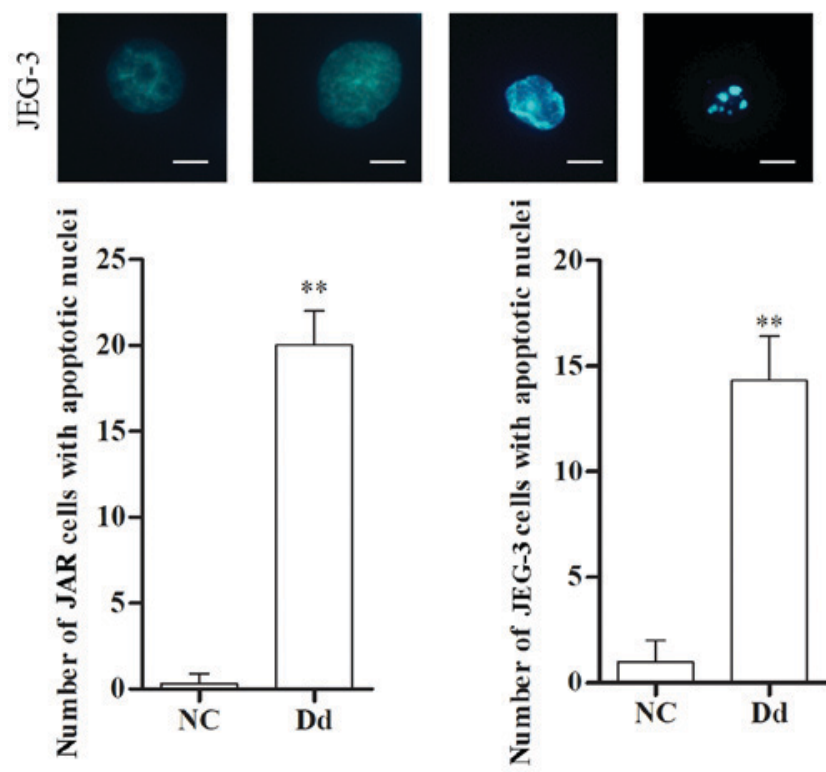

Figure 4. Morphological alterations of cell nuclei during daidzein-induced apoptosis in JAR and JEG-3 cells, observed by DAPI staining and fluorescence microscopy. Scale bar, $5 \mu \mathrm{m}$. NC: Round, intact and uniformly stained nuclei. Phase I: Rippled or creased nuclei, with chromatin mildly condensed. Phase IIa: Chromatin highly condensed. Phase IIb: Fragmented nuclei. NC, negative control; Dd, $100 \mu \mathrm{M}$ daidzein. ${ }^{* *} \mathrm{P}<0.01$ vs. the $\mathrm{NC}$ group.

western blotting. In JAR cells (Fig. 5Aa), levels of cleaved-PARP (all $\mathrm{P}<0.05)$ and cleaved caspase-3 $(25 \mu \mathrm{M}, \mathrm{P}<0.05$ vs. control;
50 and $100 \mu \mathrm{M}, \mathrm{P}<0.01$ vs. control) increased following treatment with daidzein, compared with untreated cells (Fig. 5Ab and c), indicating the induction of apoptosis. Furthermore, the levels of cleaved caspase- 9 increased following treatment with daidzein, whereas the ratio of Bcl-2 to Bax decreased, also in a dose-dependent manner (all $\mathrm{P}<0.01$; Fig. 5Ad and e). Similar results were determined in JEG-3 cells (Fig. 5Ba), where following treatment with daidzein, activation of caspase-9, caspase-3 and PARP, and a decrease in the Bcl-2/Bax ratio were observed (all $\mathrm{P}<0.01$; Fig. 5Bb-e). These data indicate that daidzein induces choriocarcinoma cell apoptosis via the mitochondrial apoptotic pathway.

\section{Discussion}

Choriocarcinoma is the most malignant form of gestational trophoblastic neoplasia (29). Although chemotherapy has notably improved the prognosis of patients with choriocarcinoma in the previous decades, a substantial proportion of patients develop drug-resistance or relapse, while certain patients succumb to brain or/and liver metastases (30). Disruption of cellular processes, including the induction of apoptosis, are likely to be involved tumorigenic mechanism of GTD (31). Therefore, development of treatments inducing apoptosis of choriocarcinoma cells may aid in improving the prognosis of patients with GTD.

Daidzein belongs to the family of isoflavones, and is abundant in soybeans and soy-based products; and therefore, may be easily obtained from daily dietary intake (32-34). Known as a type of phytoestrogen, daidzein has been reported to serve an estrogen-like function in hormone-dependent cells, including prostate cells, breast cells, and Sertoli and Leydig cells of the testes (35-37). Daidzein exhibits anti-tumor activity, including a pro-apoptotic function in numerous tumor types. Daidzein has been demonstrated to 

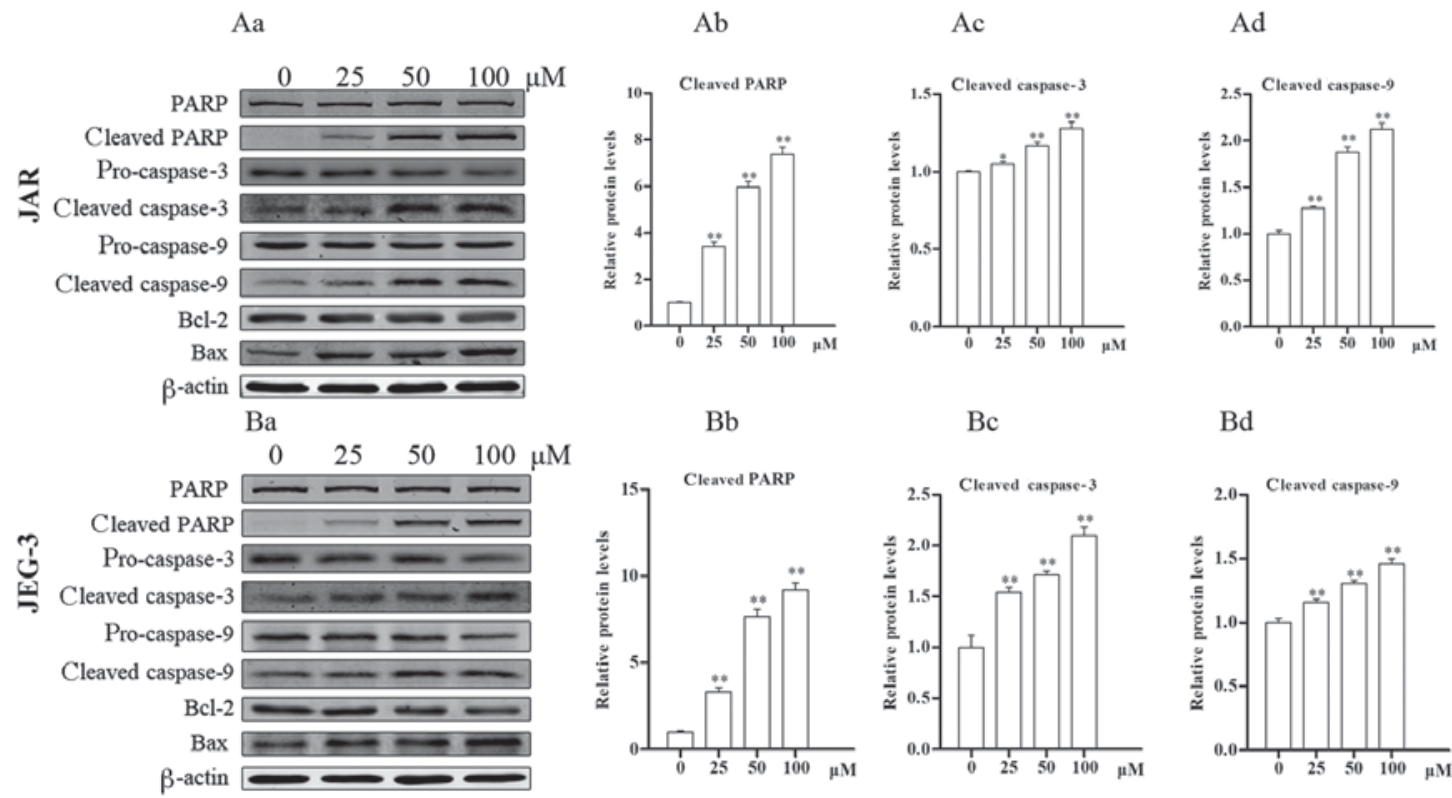

$\mathrm{Ae}$

$\mathrm{Bb}$
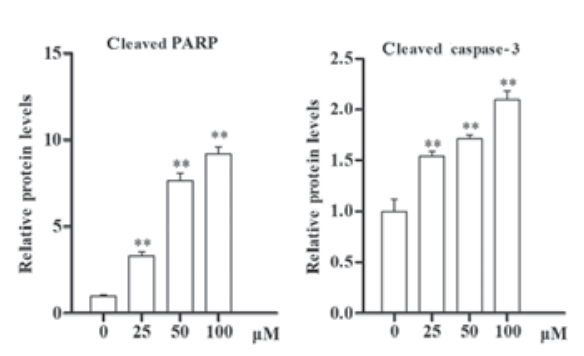

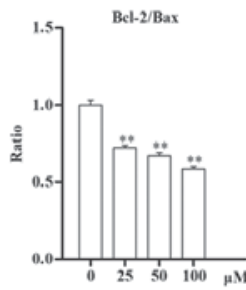

$\mathrm{Be}$

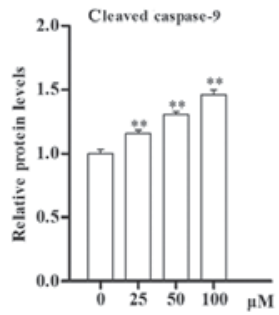

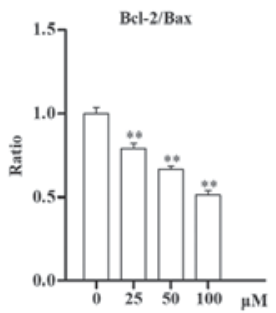

Figure 5. Effects of different concentrations of daidzein on markers of apoptosis, as determined by western blotting. Representative (Aa) western blots and quantification of apoptotic markers in JAR cells including (Ab) cleaved PARP, (Ac) cleaved caspase-3, (Ad) cleaved caspase-9 and (Ae) ratio of Bcl-2 to Bax Representative $(\mathrm{Ba})$ western blots and quantification of apoptotic markers in JEG-3 cells including (Bb) cleaved PARP, (Bc) cleaved caspase-3, (Bd) cleaved caspase-9 and $(\mathrm{Be})$ ratio of $\mathrm{Bcl}-2$ to $\mathrm{Bax} . \beta$-actin was used as internal control. ${ }^{*} \mathrm{P}<0.05$ and ${ }^{* *} \mathrm{P}<0.01$ vs. the $0 \mu \mathrm{M}$ group. Bcl-2, $\mathrm{B}$ cell lymphoma-2; Bax, Bcl-2 associated $\mathrm{X}$, apoptosis regulator; PARP, poly(ADP-ribose) polymerase 1 .

induce apoptosis in MCF-7 breast cancer cell xenografts in rodents (38). Han et al (39) demonstrated that daidzein increases the levels of reactive oxygen species and induces a decrease in mitochondrial membrane potential, leading to apoptosis induction in the BEL-7402 hepatocellular carcinoma cell line. In bladder cancer cells, daidzein also induces cell apoptosis (40).

In choriocarcinoma, daidzein regulates production of human chorionic gonadotropin (27) and, as demonstrated in the authors' previous study, also inhibits cell proliferation (28). In the present study, in vitro experiments were performed to determine whether daidzein exhibits a pro-apoptotic effect on choriocarcinoma cells.

It was demonstrated by flow cytometry that daidzein increased the percentage of early and late apoptotic JAR and JEG-3 cells, with the greatest increase observed in late apoptotic cells. The aforementioned results demonstrated a positive association between concentration of daidzein and induction of apoptosis. Immunofluorescence analysis of DAPI-stained cells indicated that alterations in nuclear morphology occurred following treatment with daidzein, with round, intact and uniformly-stained nuclei becoming rippled or creased, condensed and fragmented, indicating different degrees of apoptosis. Furthermore, the levels of cleaved-PARP and cleaved-caspase- 3 in these two cell lines significantly increased following treatment with daidzein. These results suggested that daidzein may induce choriocarcinoma cell apoptosis in a dose-dependent manner.

Daidzein induces apoptosis via the extrinsic receptor-mediated pathway, intrinsic mitochondrial pathway or endoplasmic reticulum stress pathway, depending on the type of tumor (21). For example, daidzein induces tumor necrosis factor-related apoptosis inducing ligand (TRAIL)-mediated apoptosis in prostate cancer cells (41), however activates the mitochondria-mediated pathway in breast cancer, gastric carcinoma and hepatic cancer (22-24). Vilela et al (42) reported that bio-transformed soybean extract containing daidzein increases expression of TRAIL and its receptor DR4 in melanoma, resulting in cell apoptosis. Equol, which is a metabolite of daidzein, induces apoptosis in SMMC-7721 human hepatocellular carcinoma cells through the intrinsic and endoplasmic reticulum stress pathways (26). Caspase-9 and the $\mathrm{Bcl}-2 / \mathrm{Bax}$ ratio are commonly used activity markers of the mitochondrial apoptotic pathway (43-45). In the present study, western blotting was used to detect these markers, revealing that the cleavage of caspase- 9 increased and the ratio of Bcl-2/Bax decreased in both cell lines in a dose-dependent manner, which indicated that daidzein-induced apoptosis was mediated via the mitochondrial apoptotic pathway. This process is similar to that in the human gastric carcinoma cell line BGC-823, as Tang et al (23) demonstrated that daidzein induces apoptosis via downregulation of $\mathrm{Bcl}-2 / \mathrm{Bax}$ and triggering of the mitochondrial pathway.

In conclusion, the present study demonstrated that daidzein induced choriocarcinoma cell apoptosis in a dose-dependent manner via the mitochondrial apoptotic pathway. These results provide a novel insight into the potential application of daidzein in the treatment of choriocarcinoma to improve therapeutic efficiency.

\section{Acknowledgements}

Not applicable.

\section{Funding}

The present study was supported by the National Natural Science Foundation of China (grant no. 81671491) and the 
Youth Project Fund of the First Affiliated Hospital of Xi'an Jiaotong University (grant no. 2015 YK8).

\section{Availability of data and materials}

The analyzed data sets generated during the study are available from the corresponding author on reasonable request.

\section{Authors' contributions}

WZ, RA and YX conceived and designed the study. WZ, TL and RS developed the methodology and performed the experiments. WZ and LY performed the statistical analysis. WZ and YX wrote the manuscript.

\section{Ethics approval and consent to participate}

Not applicable.

\section{Consent for publication}

Not applicable.

\section{Competing interests}

The authors declare that they have no competing interests.

\section{References}

1. Brown J, Naumann RW, Seckl MJ and Schink J: 15 years of progress in gestational trophoblastic disease: Scoring, standardization, and salvage. Gynecol Oncol 144: 200-207, 2017.

2. Berkowitz RS and Goldstein DP: Current advances in the management of gestational trophoblastic disease. Gynecol Oncol 128: 3-5, 2013.

3. Ryu N, Ogawa M, Matsui H, Usui H and Shozu M: The clinical characteristics and early detection of postpartum choriocarcinoma. Int J Gynecol Cancer 25: 926-930, 2015.

4. Alazzam M, Tidy J, Osborne R, Coleman R, Hancock BW and Lawrie TA: Chemotherapy for resistant or recurrent gestational trophoblastic neoplasia. Cochrane Database Syst Rev 12 CD008891, 2012.

5. Essel KG, Brueg1 A, Gershenson DM, Ramondetta LM, Naumann RW and Brown J: Salvage chemotherapy for gestational trophoblastic neoplasia: Utility or futility? Gynecol Oncol 146: 74-80, 2017.

6. Wong SY, Ngan HY, Chan CC and Cheung AN: Apoptosis in gestational trophoblastic disease is correlated with clinical outcome and $\mathrm{Bcl}-2$ expression but not Bax expression. Mod Pathol 12: 1025-1033, 1999.

7. Chiu PM, Ngan YS, Khoo US and Cheung AN: Apoptotic activity in gestational trophoblastic disease correlates with clinical outcome: Assessment by the caspase-related M30 CytoDeath antibody. Histopathology 38: 243-249, 2001.

8. Fong PY, Xue WC, Ngan HY, Chan KY, Khoo US, Tsao SW, Chiu PM, Man LS and Cheung AN: Mcl-1 expression in gestational trophoblastic disease correlates with clinical outcome: A differential expression study. Cancer 103: 268-276, 2005.

9. Mak VC, Lee L, Siu MK, Wong OG, Lu X, Ngan HY, Wong ES and Cheung AN: Downregulation of ASPP1 in gestational trophoblastic disease: Correlation with hypermethylation, apoptotic activity and clinical outcome. Mod Pathol 24: 522-532, 2011.

10. Braga A, Maesta I, Rocha Soares R, Elias KM, Custódio Domingues MA, Barbisan LF and Berkowitz RS: Apoptotic index for prediction of postmolar gestational trophoblastic neoplasia. Am J Obstet Gynecol 215: 336.e1-336.e12, 2016.

11. Wang TH and Wang HS: Gestational trophoblastic diseases: Current trends and perspectives. J Formos Med Assoc 94 449-457, 1995
12. Patwardhan GA, Beverly LJ and Siskind LJ: Sphingolipids and mitochondrial apoptosis. J Bioenerg Biomembr 48: 153-168, 2016.

13. Jin Z and El-Deiry WS: Overview of cell death signaling pathways. Cancer Biol Ther 4: 139-163, 2005.

14. Goldar S, Khaniani MS, Derakhshan SM and Baradaran B: Molecular mechanisms of apoptosis and roles in cancer development and treatment. Asian Pac J Cancer Prev 16: 2129-2144, 2015.

15. Kroemer G, Dallaporta B and Resche-Rigon M: The mitochondrial death/life regulator in apoptosis and necrosis. Annu Rev Physiol 60: 619-642, 1998.

16. Jiang W, Chen Y,Li B and Gao S: DBA-induced caspase-3-dependent apoptosis occurs through mitochondrial translocation of cyt-c in the rat hippocampus. Mol Biosyst 13: 1863-1873, 2017.

17. Zhang F, Yu X, Liu X, Zhou T, Nie T, Cheng M, Liu H, Dai M and Zhang B: ABT-737 potentiates cisplatin-induced apoptosis in human osteosarcoma cells via the mitochondrial apoptotic pathway. Oncol Rep 38: 2301-2308, 2017.

18. Chipuk JE, Moldoveanu T, Llambi F, Parsons MJ and Green DR: The BCL-2 family reunion. Mol Cell 37: 299-310, 2010.

19. Sheikh BY, Sarker MMR, Kamarudin MNA and Ismail A: Prophetic medicine as potential functional food elements in the intervention of cancer: A review. Biomed Pharmacother 95: 614-648, 2017.

20. Liggins J, Mulligan A, Runswick S and Bingham SA: Daidzein and genistein content of cereals. Eur J Clin Nutr 56: 961-966, 2002 .

21. Adjakly M, Ngollo M, Boiteux JP, Bignon YJ, Guy L and Bernard-Gallon D: Genistein and daidzein: Different molecular effects on prostate cancer. Anticancer Res 33: 39-44, 2013.

22. Jin S, Zhang QY, Kang XM, Wang JX and Zhao WH: Daidzein induces MCF-7 breast cancer cell apoptosis via the mitochondrial pathway. Ann Oncol 21: 263-268, 2010.

23. Tang S, Hu J, Meng Q, Dong X, Wang K, Qi Y, Chu C, Zhang X and Hou L: Daidzein induced apoptosis via down-regulation of $\mathrm{Bcl}-2 / \mathrm{Bax}$ and triggering of the mitochondrial pathway in BGC-823 cells. Cell Biochem Biophys 65: 197-202, 2013.

24. Park HJ, Jeon YK, You DH and Nam MJ: Daidzein causes cytochrome c-mediated apoptosis via the Bcl-2 family in human hepatic cancer cells. Food Chem Toxicol 60: 542-549, 2013.

25. Lo YL: A potential daidzein derivative enhances cytotoxicity of epirubicin on human colon adenocarcinoma Caco-2 cells. Int J Mol Sci 14: 158-176, 2012.

26. Liang XL, Li M, Li J and Wang XL: Equol induces apoptosis in human hepatocellular carcinoma SMMC-7721 cells through the intrinsic pathway and the endoplasmic reticulum stress pathway. Anticancer Drugs 25: 633-640, 2014.

27. Jeschke U, Briese V, Richter DU, Bruer G, Plessow D, Waldschläger J, Mylonas I and Friese K: Effects of phytoestrogens genistein and daidzein on production of human chorionic gonadotropin in term trophoblast cells in vitro. Gynecol Endocrinol 21: 180-184, 2005.

28. Zheng W, Sun R, Yang L, Zeng X, Xue Y and An R: Daidzein inhibits choriocarcinoma proliferation by arresting cell cycle at G1 phase through suppressing ERK pathway in vitro and in vivo. Oncol Rep 38: 2518-2524, 2017.

29. Bruce S and Sorosky J: Gestational trophoblastic disease. In: StatPearls. StatPearls Publishing StatPearls Publishing LLC., Treasure Island (FL), 2017.

30. Seckl MJ, Sebire NJ and Berkowitz RS: Gestational trophoblastic disease. Lancet 376: 717-729, 2010.

31. Li HW, Tsao SW and Cheung AN: Current understandings of the molecular genetics of gestational trophoblastic diseases. Placenta 23: 20-31, 2002.

32. Laurenz R, Tumbalam P, Naeve S and Thelen KD: Determination of isoflavone (genistein and daidzein) concentration of soybean seed as affected by environment and management inputs. J Sci Food Agric 97: 3342-3347, 2017.

33. Jin X, Sun J, Yu B, Wang Y, Sun WJ, Yang J, Huang SH and Xie WL: Daidzein stimulates osteogenesis facilitating proliferation, differentiation, and antiapoptosis in human osteoblast-like MG-63 cells via estrogen receptor-dependent MEK/ERK and PI3K/Akt activation. Nutr Res 42: 20-30, 2017.

34. Bhattarai K, Adhikari S, Fujitani $M$ and Kishida T: Dietary daidzein, but not genistein, has a hypocholesterolemic effect in non-ovariectomized and ovariectomized female Sprague-Dawley rats on a cholesterol-free diet. Biosci Biotechnol Biochem 81: 1805-1813, 2017.

35. Koo J, Cabarcas-Petroski S, Petrie JL, Diette N, White RJ and Schramm L: Induction of proto-oncogene BRF2 in breast cancer cells by the dietary soybean isoflavone daidzein. BMC Cancer 15: 905, 2015. 
36. Zhu Y, Xu H, Li M, Gao Z, Huang J, Liu L, Huang X and Li Y: Daidzein impairs Leydig cell testosterone production and Sertoli cell function in neonatal mouse testes: An in vitro study. Mol Med Rep 14: 5325-5333, 2016.

37. Zhang Q, Feng H, Qluwakemi B, Wang J, Yao S, Cheng G, Xu H, Qiu H, Zhu L and Yuan M: Phytoestrogens and risk of prostate cancer: An updated meta-analysis of epidemiologic studies. Int J Food Sci Nutr 68: 28-42, 2017.

38. Liu X, Suzuki N, Santosh Laxmi YR, Okamoto Y and Shibutani S: Anti-breast cancer potential of daidzein in rodents. Life Sci 91: 415-419, 2012.

39. Han BJ, Li W, Jiang GB, Lai SH, Zhang C, Zeng CC and Liu YJ: Effects of daidzein in regards to cytotoxicity in vitro, apoptosis, reactive oxygen species level, cell cycle arrest and the expression of caspase and Bcl-2 family proteins. Oncol Rep 34: 1115-1120, 2015.

40. He Y, Wu X, Cao Y, Hou Y, Chen H, Wu L, Lu L, Zhu W and $\mathrm{Gu}$ Y: Daidzein exerts anti-tumor activity against bladder cancer cells via inhibition of FGFR3 pathway. Neoplasma 63: 523-531, 2016.

41. Szliszka E and Krol W: Soy isoflavones augment the effect of TRAIL-mediated apoptotic death in prostate cancer cells. Oncol Rep 26: 533-541, 2011.
42. Vilela FM, Syed DN, Chamcheu JC, Calvo-Castro LA, Fortes VS, Fonseca MJ and Mukhtar H: Biotransformed soybean extract (BSE) inhibits melanoma cell growth and viability in vitro: Involvement of nuclear factor-kappa B signaling. PLoS One 9: e103248, 2014

43. Li J, Zhao L, Zhao X, Wang P, Liu Y and Ruan J: Foxol attenuates NaF-induced apoptosis of LS8 cells through the JNK and mitochondrial pathways. Biol Trace Elem Res 181: 104-111, 2018.

44. Shi XK, Bian XB, Huang T, Wen B, Zhao L, Mu HX, Fatima S, Fan BM, Bian ZX, Huang LF and Lin CY: Azoxystrobin induces apoptosis of human esophageal squamous cell carcinoma KYSE-150 cells through triggering of the mitochondrial pathway. Front Pharmacol 8: 277, 2017.

45. Wu J, Cai Y, Li M, Zhang Y, Li H and Tan Z: Oxymatrine promotes S-Phase arrest and inhibits cell proliferation of human breast cancer cells in vitro through Mitochondria-mediated apoptosis. Biol Pharm Bull 40: 1232-1239, 2017. 